\title{
SOME THEOREMS ON SUBSEQUENCES $†$
}

\author{
HUGH J. HAMILTON
}

It is obvious that, for any real sequence for which the sum $\Sigma$ of the moduli of its elements exists and is finite, there exists a subsequence such that the modulus of the sum of its elements is not less than $\Sigma / 2$. The purpose of this paper is to formulate and investigate analogous statements for complex sequences.

Let $\mathfrak{A}$ be the class of sequences, finite or infinite, $\left\{a_{k}\right\}$ (denoted alternatively by $A$ ) of non-zero complex numbers for which $\sum\left|a_{k}\right|<\infty$, and $\left\{a_{j}^{\prime}\right\}$ (denoted alternatively by $S$ ), the general subsequence of $\left\{a_{k}\right\}$ for fixed $\left\{a_{k}\right\}$. Let $\mathfrak{B}$ be the class of sequences $\left\{b_{k}\right\}$ (denoted alternatively by $B$ ) of non-zero complex numbers for which $\sum\left|b_{k}\right|=\infty$, and $\left\{b_{j}^{\prime}\right\}$ (denoted alternatively by $T$ ), the general subsequence of $\left\{b_{k}\right\}$ for fixed $\left\{b_{k}\right\}$.

The following facts will be established: (i) Given any sequence $\left\{a_{k}\right\} \in \mathcal{A}$, there then exists a subsequence $\left\{a_{j}^{*}\right\}$ for which $\left|\sum a_{j}^{*}\right|$ $=\sup _{S}\left|\sum a_{j}^{\prime}\right|$. (ii) If $\rho \equiv \inf _{A} \max _{S}\left|\sum a_{j}^{\prime}\right| / \sum\left|a_{k}\right|$, then $\rho=1 / \pi$. (iii) No sequence $\left\{a_{k}\right\} \in \mathfrak{U}$ exists for which $\max _{S}\left|\sum a_{j}^{\prime}\right| / \sum\left|a_{k}\right|=\rho$. (iv) Given any sequence $\left\{b_{k}\right\} \in \mathfrak{B}$, there exists a subsequence $\left\{b_{i}^{*}\right\}$ such that $\$$

$$
\begin{aligned}
& \lim \sup \left|\sum^{\prime} b_{j}^{*}\right| / \sum_{1}^{N}\left|b_{k}\right|=\sup _{T} \limsup _{N}\left|\sum^{\prime} b_{j}^{\prime}\right| / \sum_{1}^{N}\left|b_{k}\right| \\
& =\lim \sup _{N} \sup _{T}\left|\sum^{\prime} b_{j}^{\prime}\right| / \sum_{1}^{N}\left|b_{k}\right|=\lim _{N} \sup _{T} \max _{T}\left|\sum^{\prime} b_{j}^{\prime}\right| / \sum_{1}^{N}\left|b_{k}\right| .
\end{aligned}
$$

(v) If $\sigma=\inf _{B} \max _{T} \lim \sup _{N}\left|\sum^{\prime} b_{j}^{\prime}\right| / \sum_{1}^{N}\left|b_{k}\right|$, then $\sigma=\rho$. (vi)There exists a sequence $\left\{b_{k}\right\} \in \mathscr{B}$ for which $\max _{T} \lim \sup _{N}\left|\sum^{\prime} b_{j}^{\prime}\right| / \sum_{1}^{N}\left|b_{k}\right|=\sigma$.

Use will be made of abbreviations of the following sort: $A_{k} \equiv\left|a_{k}\right|$, $\phi_{k} \equiv \arg a_{k}$. For definiteness, the function "arg" will mean, throughout this paper, principal argument. Given any sequence $\left\{a_{k}\right\} \in \mathfrak{A}$, define

$$
\begin{aligned}
F(\phi) & \equiv \sum_{\cos \left(\phi-\phi_{k}\right)>0} A_{k} \cos \left(\phi-\phi_{k}\right) \\
& =\sum A_{k}\left\{\cos \left(\phi-\phi_{k}\right)+\left|\cos \left(\phi-\phi_{k}\right)\right|\right\} / 2, \quad 0 \leqq \phi \leqq 2 \pi .
\end{aligned}
$$

$\dagger$ Presented to the Society, November 27, 1937.

$\ddagger$ The notation $\sum^{\prime}$ indicates summation over precisely those elements of the subsequence which occur among the elements of the original sequence summed elsewhere in the formula. 
Being continuous, $F(\phi)$ attains its supremum. In what follows, to and including Theorem 3, $\left\{a_{k}\right\}$ will signify an arbitrary but fixed sequence of class $\mathfrak{A}$.

Theorem 1. Let $\phi^{*}$ be such that $F\left(\phi^{*}\right)=\max F(\phi)$, and let $\left\{a_{j}^{*}\right\}$ be the sequence of those elements of $\left\{a_{k}\right\}$ for which $\cos \left(\phi^{*}-\phi_{k}\right)>0$. Then $\sup _{S}\left|\sum a_{j}^{\prime}\right|=F\left(\phi^{*}\right)=\left|\sum a_{j}^{*}\right|$.

Proof. Let $\left\{a_{j}^{\prime}\right\}$ be any subsequence of $\left\{a_{k}\right\}$, and define $\phi=\arg \sum a_{j}^{\prime}$. Then

$$
\begin{aligned}
\left|\sum a_{j}^{*}\right| & \geqq \sum A_{j}^{*} \cos \left(\phi^{*}-\phi_{j}^{*}\right)=F\left(\phi^{*}\right) \geqq F(\phi) . \\
& =\sum_{\cos \left(\phi-\phi_{k}\right)>0} A_{k} \cos \left(\phi-\phi_{k}\right) \geqq \sum A_{j}^{\prime} \cos \left(\phi-\phi_{j}^{\prime}\right)=\left|\sum a_{j}^{\prime}\right| .
\end{aligned}
$$

This establishes (i).

CoROLlaRY 1.1. In the notation of Theorem $1, \phi^{*}=\arg \sum a_{j}^{*}$.

Proof. Taking $\left\{a_{j}^{\prime}\right\} \equiv\left\{a_{j}^{*}\right\}$ in the inequalities of Theorem 1 , we see that $\left|\sum a_{j}^{*}\right|=\sum A_{j}^{*} \cos \left(\phi^{*}-\phi_{j}^{*}\right)$. That is, the modulus of $\sum a_{j}^{*}$ is equal to that of its projection on the ray of angle $\phi^{*}$.

The following theorem and its corollary provide a sort of converse or dual of Theorem 1 and Corollary 1.1:

THEOREM 2. Let $\left\{\bar{a}_{j}\right\}$ be a subsequence of $\left\{a_{k}\right\}$ for which $\left|\sum \bar{a}_{j}\right|$ $=\max _{S}\left|\sum a_{j}^{\prime}\right|$, and let $\bar{\phi} \equiv \arg \sum \bar{a}_{j}$. Then $\max F(\phi)=\left|\sum \bar{a}_{j}\right|=F(\bar{\phi})$.

Proof. Let $\phi$ be any angle, $(0 \leqq \phi \leqq 2 \pi)$, and $\left\{a_{j}^{\prime}\right\}$ the sequence of those elements of $\left\{a_{k}\right\}$ for which $\cos \left(\phi-\phi_{k}\right)>0$. Then

$$
\begin{aligned}
F(\bar{\phi}) & =\sum_{\cos \left(\bar{\phi}-\phi_{k}\right)>0} A_{k} \cos \left(\bar{\phi}-\phi_{k}\right) \geqq \sum \bar{A}_{j} \cos \left(\bar{\phi}-\bar{\phi}_{j}\right) \\
& =\left|\sum \bar{a}_{j}\right| \geqq\left|\sum a_{j}^{\prime}\right| \geqq \sum_{\cos \left(\phi-\phi_{k}\right)>0} A_{k} \cos \left(\phi-\phi_{k}\right)=F(\phi) .
\end{aligned}
$$

COROLlaRY 2.1. In the notation of Theorem $2,\left\{\bar{a}_{j}\right\}$ is the sequence of those elements of $\left\{a_{k}\right\}$ for which $\cos \left(\bar{\phi}-\phi_{k}\right)>0$.

Proof. Taking $\phi=\bar{\phi}$ in the inequalities of Theorem 2, we see that

$$
\sum_{\cos \left(\bar{\phi}-\phi_{k}\right)>0} A_{k} \cos \left(\bar{\phi}-\phi_{k}\right)=\sum \bar{A}_{j} \cos \left(\bar{\phi}-\bar{\phi}_{j}\right) .
$$

In conjunction with Theorem 3 (below), this proves the assertion.

THEOREM 3. In the notation of Theorem 2, there exists no element $a_{\kappa}$ of $\left\{a_{\kappa}\right\}$ for which $\cos \left(\bar{\phi}-\phi_{\kappa}\right)=0$. 
PROOF. If there were such an element, then $\left|\sum \bar{a}_{j} \pm a_{\kappa}\right|>\left|\sum \bar{a}_{j}\right|$, so that addition of $a_{k}$ to $\left\{\bar{a}_{j}\right\}$, if it were not already therein contained, or removal of it, if it were, would provide a subsequence of $\left\{a_{k}\right\}$ to establish that $\left|\sum \bar{a}_{j}\right|<\max _{S}\left|\sum a_{j}^{\prime}\right|$, contrary to the definition of $\left\{\bar{a}_{j}\right\}$.

THEOREM 4. $\rho=1 / \pi$.

Proof. First,

$$
\int_{0}^{2 \pi} F(\phi) d \phi=2 \sum A_{k} .
$$

Thus $\max F(\phi) \geqq \sum A_{k} / \pi$, whence, by Theorem $1, \rho \geqq 1 / \pi$. To show that $\rho \leqq 1 / \pi$, consider the sequence over $\nu$ of particular finite sequences $\left\{{ }_{\nu} a_{k}\right\}$, where ${ }_{\nu} a_{k} \equiv \exp \{k \pi i /(2 \nu+1)\},(k=-2 \nu,-2 \nu+1, \cdots, 0,1$, $\cdots, 2 \nu, 2 \nu+1)$. By Corollary 2.1, for given $\nu$ any subsequence $\left\{{ }_{\nu} a_{j}^{\prime}\right\}$ of $\left\{{ }_{\nu} a_{k}\right\}$ the sum of whose elements is of maximum modulus consists of those elements whose arguments lie in a certain sector of aperture $\pi$. By the symmetry of the sequence $\left\{{ }_{\nu} a_{k}\right\}$, the midray of such a sector must lie either on a vector ${ }_{\nu} a_{k}$ or midway between two such vectors which are adjacent. In the latter case, however, Theorem 3 would be violated. Hence the former must obtain, and thus those elements of $\left\{{ }_{\nu} a_{k}\right\}$ for which $-\pi / 2<k \pi /(2 \nu+1)<\pi / 2$ constitute a subsequence the sum of whose elements is of maximum modulus. Hence, if $S(\nu)$ denotes the general subsequence $\left\{{ }_{\nu} a_{j}^{\prime}\right\}$ of $\left\{{ }_{\nu} a_{k}\right\}$,

$$
\begin{aligned}
\max _{\mathbf{S}(\nu)}\left|\sum_{j}{ }_{\nu} a_{j}^{\prime}\right| / \sum_{k}{ }_{\nu} A_{k} & =\sum_{k=-\nu}^{\nu} \cos \{k \pi /(2 \nu+1)\} /\{2(2 \nu+1)\} \\
& =1 /\{2(2 \nu+1) \sin [\pi /\{2(2 \nu+1)\}]\} ;
\end{aligned}
$$

and, as $\nu \rightarrow \infty$, this tends monotonely to $1 / \pi$. This establishes (ii).

THEOREM 5. There exists no sequence $\left\{a_{k}\right\} \in \mathfrak{A}$ for which $F(\phi)$ is constant.

Proof. If there were such a sequence $\left\{a_{k}\right\}$ then, by Theorem 1 , for each $\phi$ the sequence $\left\{a_{j}^{*}\right\}$ of those elements of $\left\{a_{k}\right\}$ for which $\cos \left(\phi-\phi_{k}\right)>0$ would be such that $\left|\sum a_{j}^{*}\right|=\max _{S}\left|\sum a_{j}^{\prime}\right|$. Hence, by Corollary 1.1 and Theorem 3 , there would exist no non-zero element of $\left\{a_{k}\right\}$, contrary to the definition of $\mathfrak{A}$.

THEOREM 6. Given an arbitrary sequence, finite or infinite, of pairs $\left(C_{k}, \psi_{k}\right)$, where the $\psi_{k}$ are real numbers and the $C_{k}$ positive numbers with $\sum C_{k}<\infty$, then $\Phi(\phi) \equiv \sum C_{k}\left|\cos \left(\phi-\psi_{k}\right)\right|$ is not constant. 
Proof. The sequence $\left\{a_{k}\right\}$ defined thus: $a_{2 k-1} \equiv C_{k} \exp \left(i \psi_{k}\right)$, $a_{2 k} \equiv C_{k} \exp \left[i\left(\psi_{k}-\pi\right)\right]$, is of class $\mathfrak{A}$, and

$$
\begin{aligned}
F(\phi) & =\sum_{\cos \left(\phi-\psi_{k}\right)>0} C_{k} \cos \left(\phi-\psi_{k}\right)+\sum_{\cos \left(\phi-\psi_{k}\right)<0} C_{k} \cos \left(\phi+\pi-\psi_{k}\right) \\
& =\sum C_{k}\left|\cos \left(\phi-\psi_{k}\right)\right|=\Phi(\phi) .
\end{aligned}
$$

The conclusion now follows from Theorem 5 .

THEOREM 7. There exists no sequence $\left\{a_{k}\right\} \in \mathfrak{A}$ for which it is true that $\max _{S}\left|\sum a_{i}^{\prime}\right| / \sum A_{k}=\rho$.

Proof. If there were such a sequence $\left\{a_{k}\right\}$, then, by Theorem 1, $F(\phi) \leqq \rho \sum A_{k}$ for all $\phi$. Hence

$$
\begin{aligned}
\int_{0}^{2 \pi}\left|\rho \sum A_{k}-F(\phi)\right| d \phi & =\int_{0}^{2 \pi}\left\{\rho \sum A_{k}-F(\phi)\right\} d \phi \\
& =2 \sum A_{k}-2 \sum A_{k}=0 .
\end{aligned}
$$

By continuity, then, $F(\phi)=\rho \sum A_{k}$ for all $\phi$. But by Theorem 5 this is impossible. This establishes (iii).

Lemma 8.1. Let $X$ be an aggregate of elements $x$ of any sort, and $\left\{f_{N}\right\}$ any sequence of functionals over $X$. Then $\sup _{x} \lim \sup _{N} f_{N}(x)$ $\leqq \lim \sup _{N} \sup _{x} f_{N}(x)$.

PRoof. For each $N$ and for all $x, f_{N}(x) \leqq \sup _{x} f_{N}(x)$. Hence, for all $x$, $\lim \sup _{N} f_{N}(x) \leqq \lim \sup _{N} \sup _{x} f_{N}(x)$, and the conclusion follows.

REMARK. Equality in the conclusion of Lemma 8.1 is not implied by the hypotheses. For, if we let $X$ represent the totality of real numbers and define $f_{N}(1 / N)=1, f_{N}(x)=0$ for $x \neq 1 / N,(N=1,2, \cdots)$, it follows that $\lim \sup _{N} f_{N}(x)=0$ for each $x$, so that $\sup _{x} \lim \sup _{N} f_{N}(x)$ $=0$, whereas $\sup _{x} f_{N}(x)=1$ for each $N$, so that lim $\sup _{\mathrm{N}} \sup _{x} f_{N}(x)=1$.

THEOREM 8. Let $\left\{b_{k}\right\} \in \mathfrak{B}$ be arbitrary. Then there exists a subsequence $\left\{b_{j}^{*}\right\}$ of $\left\{b_{k}\right\}$ for which

$$
\begin{aligned}
& \limsup _{N}\left|\sum^{\prime} b_{j}^{*}\right| / \sum_{1}^{N} B_{k}=\sup _{T} \limsup _{N}\left|\sum^{\prime} b_{j}^{\prime}\right| / \sum_{1}^{N} B_{k} \\
& \quad=\lim \sup _{N} \sup _{T}\left|\sum^{\prime} b_{j}^{\prime}\right| / \sum_{1}^{N} B_{k}=\limsup _{N} \max _{T}\left|\sum^{\prime} b_{j}^{\prime}\right| / \sum_{1}^{N} B_{k} .
\end{aligned}
$$

Proof. By Theorem 1, for each $N$ there exists a subsequence $\left\{b_{j}^{(N)}\right\}$ of $\left\{b_{k}\right\}$ for which $\left|\sum^{\prime} b_{j}^{(N)}\right| / \sum_{1}^{N} B_{k}=\sup _{T}\left|\sum^{\prime} b_{j}^{\prime}\right| / \sum_{1}^{N} B_{k}$. Let $\{N(\nu)\},(\nu=1,2, \cdots)$, be a subsequence of $\{N\}$ such that 


$$
\lim \left|\sum_{j}^{\prime} b_{j}\right| / \sum_{1}^{N(\nu)} B_{k}=\underset{N}{\lim \sup }\left|\sum_{j}^{\prime} b_{j}^{(N)}\right| / \sum_{1}^{N} B_{k},
$$

and such that

$$
\sum_{1}^{N(\nu-1)} B_{k} / \sum_{1}^{N(\nu)} B_{k}<1 / 2^{\nu+1}
$$

where the notation ${ }_{\nu} b_{j}$ represents $b_{j}{ }^{(N)}$ with $N=N(\nu)$. Define the subsequence $\left\{b_{i}^{*}\right\}$ of $\left\{b_{k}\right\}$ in such a manner that its elements coincide in order with those of $\left\{{ }_{\nu} b_{j}\right\}$ in the subscript interval (with respect to the original sequence $\left.\left\{b_{k}\right\}\right) N(\nu-1)<k \leqq N(\nu)$ for all $\nu,(N(0) \equiv 0)$. Now

$$
\limsup _{N}\left|\sum^{\prime} b_{i}^{*}\right| / \sum_{1}^{N} B_{k} \geqq \lim \sup _{\nu}\left|\sum^{\prime} b_{j}^{*}\right| / \sum_{1}^{N(\nu)} B_{k}
$$

so that from the inequality

$$
\left|\sum^{\prime} b_{i}^{*}\right| / / \sum_{1}^{N(v)} B_{k} \geqq\left|\sum_{i \downarrow}^{\prime} b_{j}\right| / / \sum_{1}^{N(\nu)} B_{k}-1 / 2^{\nu}
$$

it follows that

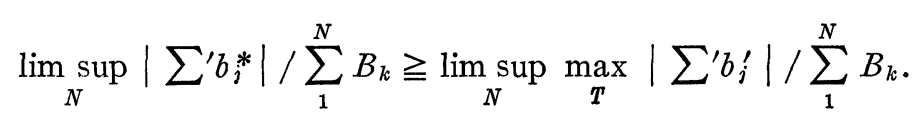

But that

$$
\underset{N}{\lim \sup }\left|\sum^{\prime} b_{j}^{*}\right| / \sum_{1}^{N} B_{k} \leqq \sup _{T} \lim _{N} \sup _{N}\left|\sum^{\prime} b_{j}^{\prime}\right| / \sum_{1}^{N} B_{k}
$$

is obvious, and that

$$
\sup _{T} \limsup _{N}\left|\sum^{\prime} b_{j}^{\prime}\right| / \sum_{1}^{N} B_{k} \leqq \limsup _{N} \max _{T}\left|\sum^{\prime} b_{j}^{\prime}\right| / \sum_{1}^{N} B_{k}
$$

follows from Lemma 8.1. The conclusion follows. This establishes (iv).

Lemma 9.1. $\sigma \geqq \rho$.

Proof. By Theorem 8,

$$
\begin{aligned}
\sigma & \equiv \inf _{B} \max _{T} \lim _{N} \sup _{N}\left|\sum^{\prime} b_{j}^{\prime}\right| / \sum_{1}^{N} B_{k} \\
& =\inf _{B} \lim _{N} \sup _{T} \max _{T}\left|\sum^{\prime} b_{j}^{\prime}\right| / \sum_{1}^{N} B_{k} \geqq \rho,
\end{aligned}
$$


which establishes the lemma.

Consider now the sequence $\left\{b_{k}^{*}\right\}$ defined thus: $b_{k}^{*} \equiv e^{i k},(k=1$, $2, \cdots)$, and define

$$
F_{N}(\phi) \equiv \sum_{1}^{N}\{\cos (\phi-k)+|\cos (\phi-k)|\} / 2 N, \quad 0 \leqq \phi \leqq 2 \pi .
$$

LEMMA 9.2. $\operatorname{Lim}_{N} \operatorname{Osc}_{\phi} F_{N}(\phi)=0$.

Proof. Let $\epsilon>0$ be arbitrary; let $K$ be such that, for each $\phi$, $\phi \equiv p(\phi)+\eta_{\phi}(\bmod 2 \pi)$ for some $\eta_{\phi}$ for which $\left|\eta_{\phi}\right|<\epsilon$ and some integer $p(\phi)$ for which $0 \leqq p(\phi) \leqq K$; and let $N$ be such that $K / N<\epsilon$. Then, for each $\phi$,

$$
\begin{aligned}
\left|F_{N}(\phi)-F_{N}(0)\right| \leqq & \mid \sum_{k=1}^{N-p(\phi)}\left\{\cos \left(k-\eta_{\phi}\right)\right. \\
& \left.+\left|\cos \left(k-\eta_{\phi}\right)-\cos k-\right| \cos k \mid\right\}|| / 2 N \\
& +\left|\sum_{k=1}^{p(\phi)}\{\cos (\phi-k)+|\cos (\phi-k)|\}\right| / 2 N \\
& +\left|\sum_{N-p(\phi)+1}^{N}\{\cos k+|\cos k|\}\right| / 2 N<3 \epsilon .
\end{aligned}
$$

This establishes the lemma.

LEMma 9.3. $\operatorname{Lim}_{N} F_{N}(\phi)=\rho$ uniformly in $\phi$.

Proof. The assertion follows from Lemma 9.2 and the fact that, for each $N, \int_{0}^{2 \pi} F_{N}(\phi) d \phi=2$.

THEOREM 9. $\sigma=\rho$.

Proof. Applying Theorem 2 to the (finite) sequence of those elements of $\left\{b_{k}^{*}\right\}$ for which $k \leqq N$, we find that

$$
\max _{T}\left|\sum^{\prime} b_{j}^{* \prime}\right| / \sum_{1}^{N} B_{k}^{*}=\max _{\phi} F_{N}(\phi)
$$

which tends to $\rho$, by Lemma 9.3. By Theorem 8 and Lemma 9.1, this establishes the theorem, and hence also (v).

THEOREM 10. There exist an uncountably infinite number of subsequences $\left\{b_{j}^{*}\right\}$ of $\left\{b_{k}^{*}\right\}$ for which

$$
\lim _{N}\left|\sum^{\prime} \bar{b}_{j}^{*}\right| / \sum_{1}^{N} B_{k}^{*}=\max _{T} \lim _{N} \sup _{N}\left|\sum^{\prime} b_{j}^{* \prime}\right| / \sum_{1}^{N} B_{k}^{*}=\rho=\sigma .
$$


Proof. Let $\phi^{\prime}$ be arbitrary, and let $\left\{b_{i}^{*}\right\}$ be the sequence of those elements of $\left\{b_{k}^{*}\right\}$ for which $\cos \left(\phi^{\prime}-\phi_{k}^{*}\right)>0$. Then, by inequalities like those used in the proof of Theorem 2, for each $N$,

$$
F_{N}\left(\phi^{\prime}\right) \leqq\left|\sum^{\prime} b_{j}^{*}\right| / \sum_{1}^{N} B_{k}^{*} \leqq \max _{T}\left|\sum^{\prime} b_{j}^{* \prime}\right| / \sum_{1}^{N} B_{k}^{*}=\max _{\phi} F_{N}(\phi),
$$

and the conclusion is seen to follow from Lemma 9.3 and Theorem 8 . This establishes (vi).

THEOREM 11. If $\Phi_{N}(\phi) \equiv \sum_{1}^{N}|\cos (\phi-k)| / N, \quad(0 \leqq \phi \leqq 2 \pi)$, then $\lim _{N} \Phi_{N}(\phi)=2 / \pi$ uniformly in $\phi$.

Proof. As in the proof of Lemma 9.2, it can be shown that $\lim _{N} \cos \phi \Phi_{N}(\phi)=0$. Also,

$$
\int_{0}^{2 \pi} \Phi_{N}(\phi) d \phi=4
$$

The conclusion follows.

REMARK. The sequence $\left\{b_{k}^{*}\right\}$ could equally well have been taken thus: $b_{k}^{*}=e^{i \delta k},(k=1,2, \cdots)$, where $\delta$ is any number incommensurable with $\pi$.

Pomona College 